\title{
MORPHOLOGICAL AND NANOMECHANICAL INVESTIGATIONS OF MAGNEVIST NANO-ENCAPSULATED DENDRIMERS BY ATOMIC FORCE MICROSCOPY
}

Hosam Gharib Abdelhady, College of Pharmacy, Taibah University, Al Madinah Al Munawarrah, Kingdom of Saudi Arabia, National Organization for Drug Control and research, Cairo-Egypt

Received: 1 May 2012, Accepted in revised form: 3 May 2012, Published: 29 May 2012 Corresponding author: Hosam Gharib Abdelhady, e-mail: hosamgharib@hotmail.com

\section{ABSTRCAT:}

Objectives: This research aims at investigating the effect of nano-encapsulating the Magnevist ${ }^{\mathrm{TM}}$, a magnetic resonance imaging agent, within generation four, 1, 4- diaminobutane core polyamidoamine dendrimers on their molecular morphology and their nano-mechanical properties in liquid.

Methods: Atomic force microscopy was applied in its imaging and force measuring modes to investigate, on the molecular scale, the morphological and nano-mechanical changes in generation four, 1, 4-diaminobutane core polyamidoamine dendrimers due to the nano-encapsulation of Magnevist in liquid.

Results: The weight gain of dendrimers indicates the loading of $\sim 30$ Magnevist molecules. This has increased the rigidity of the dendrimer molecules, compared to unloaded dendrimers. Atomic force microscopy showed individual well-defined nano-spherical particles with nanoscopic dimensions of $(40 \pm 13 \mathrm{~nm}$ in diameter and $4.38 \pm 0.54 \mathrm{~nm}$ in height). In contrast, imaging of non encapsulated dendrimers revealed loose aggregates of $15 \pm 3.5 \mathrm{~nm}$ in diameter and $0.9 \pm 0.2 \mathrm{~nm}$ in height.

Conclusions: The atomic force microscopy, in liquid, was successfully applied to differentiate between Magnevist nano-encapsulated and non-encapsulated dendrimers, in their morphology and in their nanomechanical properties. The results confirm the nano-encapsulation of Magnevist within generation four, 1,4diaminobutane core polyamidoamine dendrimers. This loading increased the rigidity of the nanoencapsulated dendrimer, packed $\sim 9$ Magnevist- $\mathrm{G}_{4}$ molecules together and may probably enhance the magnetic resonance images and increase their duration of time in the bloodstream when compared with Magnevist alone. Thus elongating the imaging sessions without the need for additional contrast agent doses.

Keywords: Atomic force microscopy (AFM), dendrimers, Magnevist, Magnetic resonance imaging (MRI).

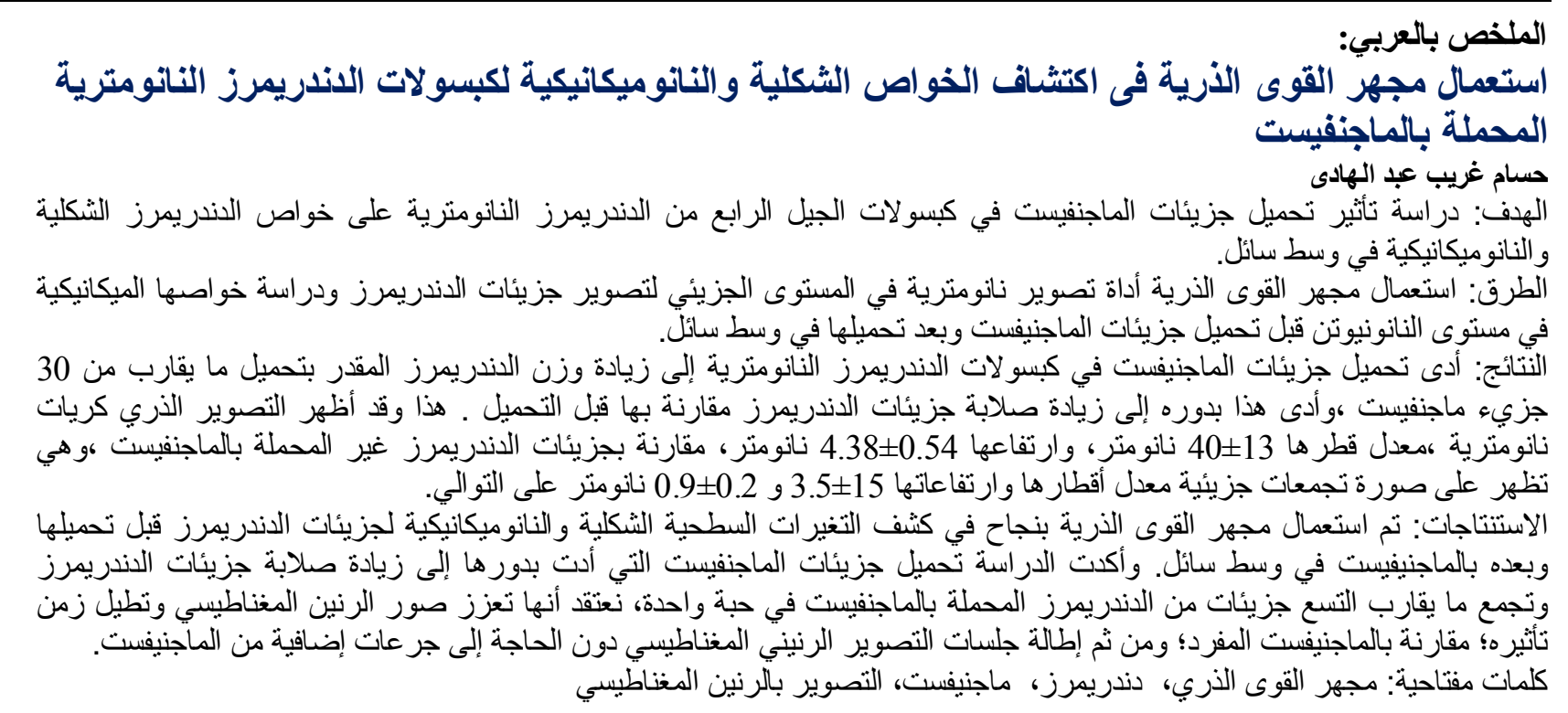

Copyright $\odot 2012$ H G Abdelhady and LJPCP. This is an open access article distributed under the Creative Commmons Attribution 3.0 License, which permits unrestricted use, distribution, and reproduction in any medium, provided the original work is properly cited. 


\section{INTRODUCTION:}

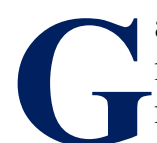
adolinium (Gd III) is a paramagnetic metal used frequently in magnetic resonance imaging (MRI) [1-2]. However, the toxicity of the free Gd III metal and its low efficiency as a contrast agent are considered as main barriers against the achievement of efficient molecular imaging. Fortunately, the rational design of the Gd III ions in water soluble chelates as diethylenetriaminepentaacetic acid (DTPA) known as (Magnevist $\left.{ }^{\circledR}\right)$, Figure 1, has overcome those barriers [3-5].

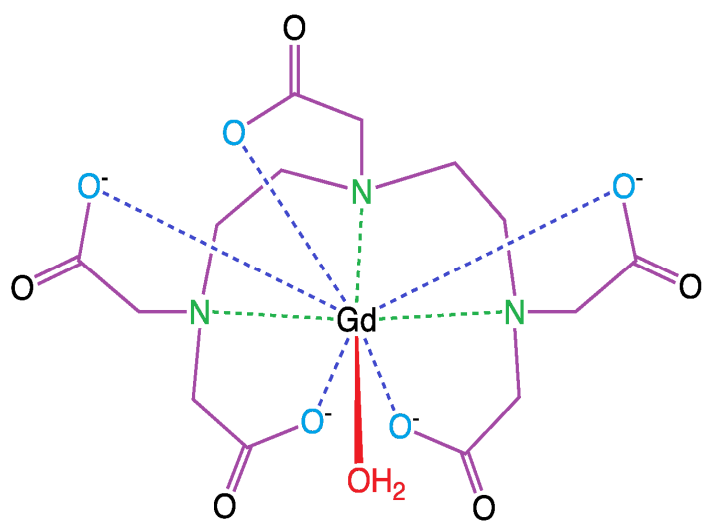

Figure1. Magnevist molecule.

However, clinically used Gd III chelates still suffer from nonspecificity, low relaxivity, rapid extravasation and rapid whole body clearance [6]. To this end, it was found that the conjugation of Gd III chelates with polyamidoamine (PAMAM) dendrimer were not only efficient and effective in prolonging intravascular retention and circulation time of Gd III chelates due to their large sizes but also effective in modulating and relaxing water protons [7-12]. Wiener E and Toth E [13-14] reported a strong increase in molecular relaxivity, which was attributed not only to the large number of Gd III-DTPA complexes attached to a single dendrimer molecule but also to a higher ionic relaxivity per Gd III. PAMAM dendrimers,Figure 2. are mono dispersed water soluble, biocompatible macromolecules with well controlled sizes, nanoscopic three dimensions and numerous surface and interior amine groups to which MRI probe scan be coupled [15-17]. These dendrimers are constructed from various initiator cores on which each complete iterative reaction sequence results in a new dendrimer "generation".

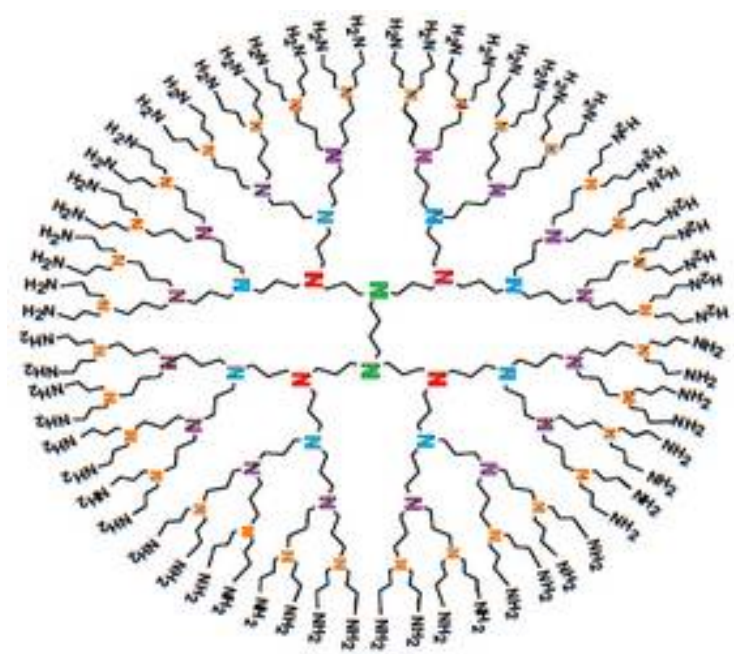

Figure2. G4 with 2 amines on the core (green) and 64 amine groups on the surface (black).

The molecular weight $(\mathrm{Mw})$ of the dendrimer is nearly doubled with the increase in generation, the number of primary amine surface groups exactly doubled and the diameter increases by $\sim 1 \mathrm{~nm}$ [18]. Generation four, 1,4-diaminobutane core PAMAM dendrimers (G4), as an example, has 64 primary amine groups, 62 tertiary amine groups and has a Mw of 14242 Dalton (Da). However, the possibility of the aggregation of contrast agent complexes may have a profound implications on its effective molecular weight, and hence on its behavior. Thus, an accurate assessment of the morphological and nano-mechanical properties of these nanocomplexes is required. To this end, atomic force microscopy (AFM) has immerged to provide an unparalleled spatial resolution of the order of angstroms and force resolution of Pico-Newton's [19]. The main advantages of AFM over other ultrahigh resolution microscopy techniques is that sample preparation is relatively simple and does not involve negative staining or shadow casting with a metal coating (as required for electron microscopy), hence AFM measurements can be made to reflect directly the natural topography and nanomechanical properties of the specimen [19-22]. A well approach to measure the nanomechanical properties of different surfaces would be to record the forcedistance curves (f-d-c(s)) and measure the adhesion forces and Young's modulus directly from the force curves [21]. The AFM tip is then ramped along the vertical axis and the cantilever deflection is acquired. An AFM f-d-c is the result of the tipsample interaction and the spring constant of the cantilever (Figure 3). The tip sample force is given by Hooke's law: $\mathrm{F}=-\mathrm{kc} \gamma \mathrm{c}$. Where $\mathrm{F}$ is the Force in 
nano Newton $(\mathrm{nN}), \gamma \mathrm{c}$ is the cantilever deflection in nanometers $(\mathrm{nm})$ and $\mathrm{kc}$ is the cantilever spring constant in $\mathrm{nN} / \mathrm{nm}$. Here, we suggest a simple and fast AFM method, in liquid, for the investigation of the morphological and nano-mechanical changes in G4 before and after their nanoencapsulation with Magnevist (Magnevist-G4).

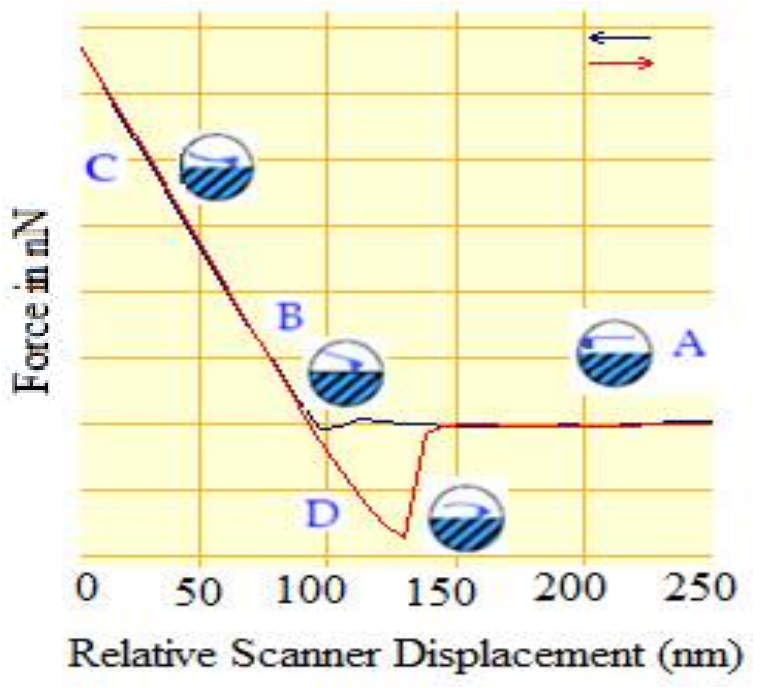

Figure3. Force-distance curves (f-d-c(s)). During a force distance sequence the tip is approaching vertically from (A) to the sample surface at a constant speed until it experiences a weak attraction force at point (B). The tip continue in approaching the surface until a predetermined set point of maximum load is reached $(\mathrm{C})$. The direction of motion is then reversed, and the tip is withdrawn from the sample surface. As the tip is withdrawn, it adheres to the sample (D). The extent of the adhesion force is then calculated from Hooke's law.

\section{MATERIALS AND METHODS: MATERIALS.}

All routine chemicals, LC-MS Grade (CHROMASOLV) solvents and Generation 4, 1, 4-diaminobutane core, PAMAM dendrimers were obtained from Sigma-Aldrich (Milwaukee, WI, USA). Deionized (DI) water was obtained from Millipore-Billerica (Massachusetts, USA) with water of resistivity of $18.2 \mathrm{M} \Omega . \mathrm{cm}-1$.

\section{PREPARATION OF PAMAM DENDRIMER- COATED MICA FOR AFM IMAGING AND FORCE MEASURING.}

Samples were obtained as methanolic solutions which were dried under a stream of nitrogen and subsequently placed in a vacuum (pressure $\sim 0.013$ mbar) for one day. The dried material was redissolved in DI water. A $0.1 \mu \mathrm{g} . \mathrm{ml}-1$ aqueous stock solution was then prepared and stored at $4{ }^{\circ} \mathrm{C}$ for a maximum of a few days. For AFM imaging, a $20 \mu \mathrm{l}$ drop of 100 femtogram $/ \mathrm{ml}$,Fg.mll. dendrimer solution was placed on a freshly cleaved mica and left for $10 \mathrm{~min}$. The substrate was then rinsed gently with DI water to remove loosely adsorbed G4 molecules. For force experiments, a $0.1 \mu \mathrm{g} . \mathrm{ml}-1 \mathrm{G} 4$ was placed onto mica and left for $10 \mathrm{~min}$. The substrate was then rinsed gently with DI water.

\section{MAGNEVIST ENCAPSULATION FOR AFM IMAGING AND FORCE MEASURING:}

Dry G4 (0.14g, $9.8 \times 10-6 \mathrm{~mol})$ was dissolved in 25 $\mathrm{ml}$ DI water to make a clear, colorless solution. Magnevist $(0.47 \mathrm{~g}, 5 \times 10-4 \mathrm{~mol})$ was then added to the dendrimer solution, and the mixture was stirred for 24 hours at room temperature. The undissolved solid was filtered. The mixture was then dialyzed against DI water for 7 hours with several water changes using a Slide-A-Lyzer Dialysis Cassettes, 7K MWCO (Thermo Scientific, Rockford, IL) and the product was lyophilized overnight. To produce Megnevist treated G4 coated mica, a $20 \mu \mathrm{l}$ drop of 200 Picogram/ml (Pg.ml-1) dendrimer solution was placed on a freshly cleaved mica and left for $10 \mathrm{~min}$. The substrate was then rinsed gently with DI water to remove loosely adsorbed molecules. For force experiments, a $0.3 \mu \mathrm{g} . \mathrm{ml}-1$ Magnivest encapsulated G4 was placed onto mica and left for $10 \mathrm{~min}$ and the substrate was then rinsed gently with DI water.

\section{AFM IMAGING EXPERIMENTS:}

All tapping mode images were obtained in DI water to partially simulate the physiological environment, using a multimode AFM, with a Nanoscope IIIa controller (Veeco, Santa Barbara, CA, USA). All experiments were performed within 1 day of sample preparation. Mica, G4 and Magnivest-G4 samples were imaged by single silicon nitride (Si3N4) probes (100 $\mu \mathrm{m}$ long V-shaped cantilevers, with nominated spring constant of $\sim 0.32 \mathrm{~N} / \mathrm{m}$, and resonant frequencies in water between 9 and $12 \mathrm{kHz}$ ). As imaging in liquids eliminates the capillary interaction between the AFM tip and the sample surface, imaging forces of $<0.1 \mathrm{nN}$ could be achieved in order to minimize any sample deformation by the probe 21 .

\section{AFM FORCE EXPERIMENTS:}

Force-distance measurements were recorded in DI water ( $\mathrm{pH}$ ca $6.4 \pm 0.1$ ) using the same Si3N4 tip for experiments on mica, G4 and G4-Magnivest. Previous studies have highlighted the sensitivity of force measurements to differences in the nanomechanical properties at the probe-sample interface $[20,22]$. Here, the AFM is used to differentiate between G4-Magnivest and G4. Curves 
were then recorded on mica to determine the background level of the interaction. The slope of the contact region of the force-distance approach curve was used to convert the measured cantilever deflection from the relative units of volt to the absolute units in $\mathrm{nm}$. The loading forces were maintained at $3.5 \mathrm{nN}$ to minimize any damage of the probe and/or surfaces during each experiment. The Young's Modulus for G4 and G4-Magnivest were calculated by the Scanning Probe Imaging Processor software Version 4.7, Image Metrology, Denmark.

\section{RESULTS AND DISCUSSION:}

The loading of Magnevist in G4 was determined based on the weight gain in dendrimers after their interaction with Magnevist and subsequent washing, dialysis and drying. The weight gain was found to be $(0.282 \mathrm{mg})$. This indicates the attachment of $\sim 30.5$ Magnevist molecules per one G4 molecule. Figure 4 (a-c) show topographic AFM images of the adsorption of G4 at the watermica interface following the exposure of mica to a $40 \mu 1$ of $100 \mathrm{fg} . \mathrm{ml}-1$ aqueous solution of G4.

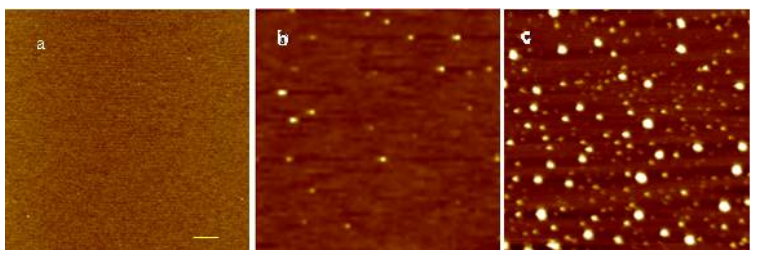

Figure4. AFM height images of (a) mica, (b) G4 dendrimers on mica, and (c) Magnevist-G4 on mica.

In contrast to the essentially featureless images of the atomically flat mica surface, Figure 4 (a), globular features were observed. The average of their measured diameters and heights were $15 \pm 3.5$ $\mathrm{nm}$ and $0.9 \pm 0.2 \mathrm{~nm}$ respectively, Figure 4 (b). The decrease in height of these dendritic structures when compared to their theoretical diameter (4.5 $\mathrm{nm}$ ) is implying the flattening of individual G4 on mica due to its open flexible behaviour. This behaviour was previously reported [22-24] where dendrimers exist as an open plate like molecules in which the dendrimer branches of neighboring molecules can inter-penetrate each other to make aggregates. These aggregates were confirmed by the analysis of their volume. On the basis of the dome shape determined from our images, a molecular volume can be calculated from the following equation: $\mathrm{V}=1 / 6 \mathrm{~h}(\mathrm{~h} 2+3 / 4 \mathrm{~d} 2)$ where $\mathrm{h}$ is the height and $\mathrm{d}$ is the diameter of the cap [25]. The number of molecules per feature was then calculated by dividing the calculated volume by the theoretical value of the individual dendrimer molecule. The average number of G4 molecules per aggregate was found to be $3.56 \pm 0.53$ molecules. Figure 4(c) shows images of Magnevist-G4 complexes adsorbed onto mica from 20 Pg.ml-1 aqueous solution. Well defined spherical structure were obtained. The average of their measured diameters and heights were $40 \pm 13$ and $4.38 \pm 0.54 \mathrm{~nm}$ respectively. Both G4 and Magnevist-G4 complexes on mica are depicted in three dimensions (3D) as shown in Figure 5 (a) and (b) respectively.

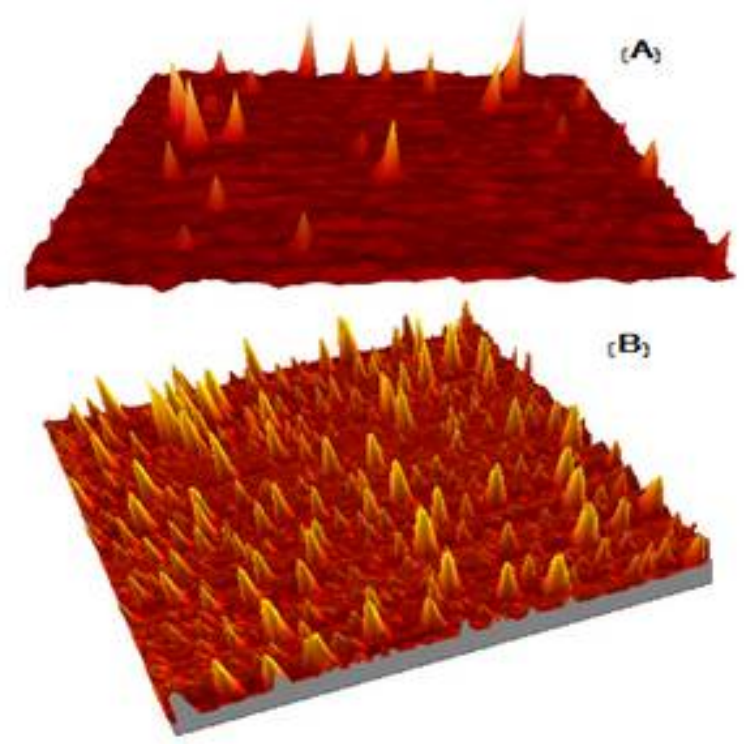

Figure5. 3D AFM images of (a) G4 and (d) MagnevistG4. All images were done in DI water. Scale bar is $170 \mathrm{~nm}$. $\mathrm{Z}$ scale $=4 \mathrm{~nm}$.

It is obvious that most of the G4 appears as small aggregates above the mica substarct while few G4 makes larger aggregates. This indicates that G4 exists in a relatively open planar structure that interpenetrates each other to make a film on mica [22]. Any extra G4 molecules then may lie upon that film and make aggregates [22-24]. In contrast, most of the magnives-G4 complexes appears as similar discreat spherical features that most likely reflect the rigidity of the complex due to the loading of Magnevist 
within G4. This loading enabled the molecules to retain their shape on mica surface and appear as discrete spherical features. Since Magnevist has carboxylic acid groups (Figure 1), it forms amine salts with both the surface primary amine and the interior tertiary amine groups of G4 [26]. Theoretically, the formation of Magnevist complexes with G4 will increase the diameter of the G4 molecule by $\sim 2 \mathrm{~nm}$ [27] and increases the volume of the complex to be $\sim 143.72 \mathrm{~nm} 3$ (considering a sphere). Experimentally, the volume analysis of the Magnevist-G4 complexes was $1221.48 \mathrm{~nm} 3$. This indicates the presence of $8.49 \pm 0.32$ molecules forming an individual MRI particle. Since most of the Magnevist-G4 complexes appear as nearly similar 3D features, thus, the packing for 9 spheres of equal sizes may be suggested as in Figure 6.

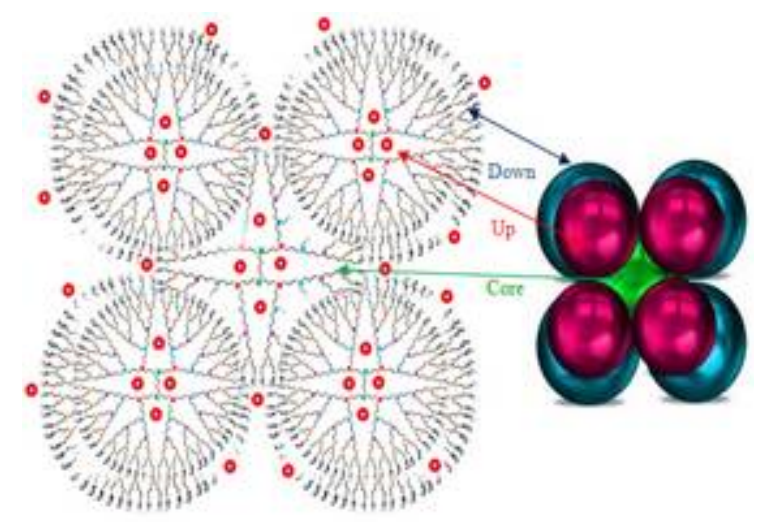

Figure6. Packing of 9 Magnevist-G4 molecules. Where one $\mathrm{G}_{4}$ molecule is shown at the core, $4 \mathrm{G}_{4}$ molecules are shown below the core and $4 \mathrm{G}_{4}$ molecules are shown Above the core. Magnivest molecules pack these $\mathrm{G}_{4}$ molecules together.

This packing increases the size and hence the contrast of the MRI complex. New et al [6] reported that G6 dendrimers has the highest increase in relaxivity based on its individual molecular size $(7.2 \mathrm{~nm}$ diameter) and surface amine number (256) if compared to smaller dendrimers. However, as the individual dendrimers size increases, its toxicity increases and its biocompatibility decreases [11]. In contrast, the attachment of $9 \mathrm{G} 4$ molecules in one particle may provide more biocompatibility by having the particle dissociated to 9 small G4 molecules and excreted via the kidney [11].
Figure 7(a-c) show representative series of $\mathrm{f}-\mathrm{d}-\mathrm{c}(\mathrm{s})$ obtained for each tip-substrate (mica (a), G4 (b) and Magnevist-G4 (c)) combinations. Within these series of measurements, it was found that the largest pull off forces was recorded on G4 surface $(0.78 \pm 0.10 \mathrm{nN})$ (Figure $7(\mathrm{~b})$ ). In the other two combinations (i.e. on Magnevist-G4 surface, Figure 7(c) and on mica Figure 7(a)), smaller pull off forces of $0.58 \pm 0.16$ and $0.10 \pm 0.02 \mathrm{nN}$ were observed respectively.

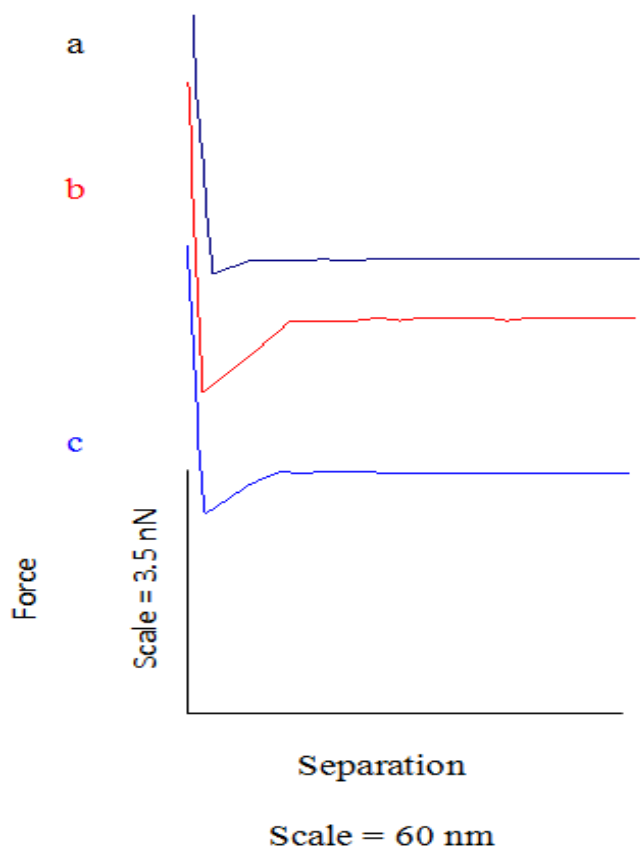

Figure7. F-d-c(s) recorded between a Silicon tip and (aMica, b-G4 and c- Magnevist-G4) in DI water.

Under conditions employed in our experiments (i.e. DI water, $\mathrm{pH}$ ca. $6.6 \pm 0.1$ ) it is therefore likely that both the G4 and Magnevist-G4 surfaces would have protonated surface primary amine groups $(\mathrm{PKa} \sim$ 10.7) [28]. Thus, the pull off forces recorded between the tip and both G4 and Magnevist-G4 surfaces could be attributed to the electrostatic attractive interactions between the available protonated primary amine groups $(\mathrm{R}-\mathrm{NH} 3)+$ and the silicon tip (behaved as silicon oxide). The decrease in the pull off forces on Magnevist-G4 $(0.58 \pm 0.16)$ surface if compared with G4 surface $(0.78 \pm 0.10 \mathrm{nN})$ is most probably attributed to the interaction of Magnevist molecules with some of the surface (R$\mathrm{NH} 3)+$ on G4. This interaction then, decreases the number of the (R-NH3)+ available for the interaction with the silicon tip during the force 
measurements. As a result, smaller pull off forces were recorded on the Magnevist-G4 surface than that recorded on the G4 surface. Another possible explanation may attributes the smaller pull off forces recorded on Magnevist-G4 to the decrease of the Tip- Magnevist-G4 interacting area, as a result of an increase in the rigidity of this system compared to the G4 system, Figure 8 shows f-d-c(s) done on G4 and Magnevist-G4 surfaces (only the approach curves are shown). The difference in rigidity was seen as differences in the slops of the force curves after the tip-sample contact, where the harder surface (Magnevist-G4) repels the tip stronger than the softer $(\mathrm{G} 4)$ surface. The yield will be a very steep slope on Magnevist-G4 complexes with a Young's modulus of 2.04 x108 Pa versus less steep slope for the G4 with a Young's modulus of 9.94 x107Pa G4.

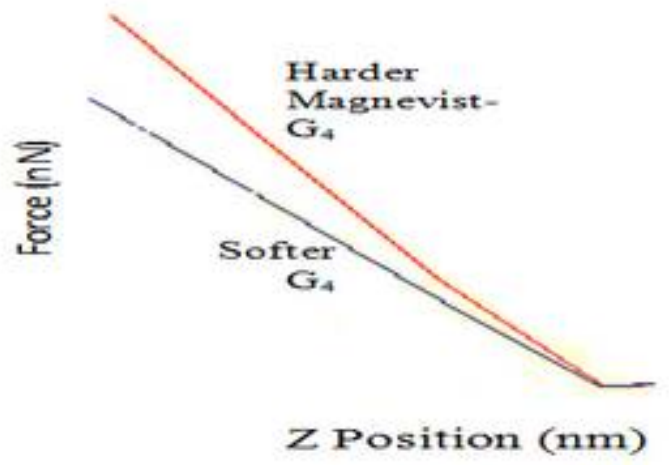

Figure8. Approach, f-d-c(s): Differences in the rigidity between G4 (flexible) and Magnevist-G4 (rigid) are shown.

\section{CONCLUSION:}

The AFM successfully monitored the loading of Magnevist ( $\geq 30$ molecules) within individual G4 molecules. The loading has increased the rigidity of the $\mathrm{G} 4$ and enhanced the physical stability of the loaded G4 spheres on mica. This observation indicates the ability of AFM imaging and force measuring to detect the loading of Magnevist within G4 molecules and to investigate its effect on the G4 morphology and nanomechanical properties in liquid.

\section{ACKNOWLEDGMENT AND FUNDING SOURCES:}

This research received no specific grant from any funding agency in the public, commercial, or not-for-profit sectors.

\section{REFERENCES:}

1) Lauffer RB. Paramagnetic Metal Complexes as Water Proton Relaxation Agents for NMR Imaging: Theory and Design. Chem Rev. 1987; 87: 901-927.

2) Lide, DR, ed. CRC Handbook of Chemistry and Physics. 86th ed. Florida: CRC Press; 2005.

3) Caravan P, Ellison JJ, McMurray TJ, Lauffer RB. Gadolinium(III) Chelates as MRI Contrast Agents: Structure, Dynamics, and Applications. Chem Rev. 1999; 99: 2293-2352.
4) Goyen
M, Lauenstein
TC, Herborn CU, Debatin JF, Bosk S, Ruehm SG. 0.5 M Gd chelate (Magnevist) Versus 1.0 M Gd III Chelate (Gadovist): Dose-Independent Effect on Image Quality of Pelvic Three-Dimensional MRAngiography. J Magn Reson Imaging; 2001; 14(5):602-607.

5) Raymond K N, Pierre VC. Next Generation, High Relaxivity Gadolinium MRI Agents. Bioconjugate Chem. 2005; 16: 3-8.

6) Nwe K, Bryant LH Jr, Brechbiel MW. Poly(amidoamine) Dendrimer Based MRI Contrast Agents Exhibiting Enhanced Relaxivities Derived via Metal Pre-ligation Techniques. Bioconjug Chem. 2010; 21(6): 1014-1017.

7) Bryant $\mathrm{LH} \mathrm{Jr}$, Brechbiel $\mathrm{MW}$, Wu C, Bulte JWM, Herynek V, Frank JA. Synthesis and Relaxometry of High-Generation $(\mathrm{G}=5,7,9$ and 10$)$ PAMAM Dendrimer DOTA-Gadolinium Chelates. J Magn Res Imaging. 1999; 9:348-352.

8) Kobayashi $\mathrm{H}$, Brechbiel MW. Nano-sized MRI Contrast Agents with Dendrimer Cores. Adv Drug Deliv Rev. 2005; 57(15):2271-86.

9) Luo K, Liu G, She W, Wang Q, Wang G, He $\mathrm{B}$, et al. Gadolinium-Labeled Peptide Dendrimers with Controlled Structures as Potential Magnetic Resonance Imaging Contrast Agents. Biomaterials. 2011; 32: 7951-7960.

10) Polasek M, Hermann P, Peters JA, Geraldes, CFGC, Lukes I. PAMAM Dendrimers Conjugated with an Uncharged Gadolinium(III) Chelate with a Fast Water Exchange: The Influence of Chelate Charge on Rotational Dynamics. Bioconjugate Chem. 2009; 20: 21422153.

11) Tomalia DA, Reyna LA, Svenson S. Dendrimers as Multi-Purpose Nanodevices for Oncology Drug Delivery and Diagnostic Imaging. Biochem Soc Trans. 2007; 35: 61-67.

12) Wang SJ, Brechbiel M, Wiener EC. Characteristics of a New MRI Contrast Agent Prepared From Polypropyleneimine Dendrimers, Generation 2. Invest Radiol. 2003; 38:662-668. 
13) Wiener E, Brechbiel MW, Brothers $\mathrm{H}$, Magin RL, Gansow OA, Tomalia DA, Lauterbur PC. Dendrimer-Based Metal Chelates: A New Class of Magnetic Resonance Imaging Contrast Agents. Magn Reson Med. 1994; 31:1-8.

14) Toth E, Pubanz D, Vauthey S, Helm L, Merbach AE. The Role of Water Exchange in Attaining Maximum Relaxivities for Dendrimeric MRI Contrast Agents. Chem Eur J. 1996; 2: 16071615.

15) Tomalia DA, Baker H, Dewald J, Hall M, Kallos G, Martin S, Roeck J, Ryder J, Smith PA. New Class of Polymers: Starburst Dendritic Macromolecules. PJ. 1985; 17: 117-132.

16) Tomalia DA, Baker H, Dewald J, Hall M, Kallos G, Mar-Tin S, Roeck J, Ryder J, Smith P. Dendritic Macromolecules: Synthesis of Starburst Dendrimers. Macromolecules. 1986; 19: 24662468.

17) Tomalia DA, Fréchet JM. Introduction to the Dendritic State. Dendrimers and Other Dendritic Polymers. 1st ed. New York: John Wiley \& Sons Inc; 2001.

18) Ottaviani MF, Matteini PMB, Turro NJ, Jockusch S, Tomalia DA. Characterization of Starburst Dendrimers and Vesicle Solutions and Their Interactions by $\mathrm{Cw}$ - and Pulsed-Epr, Tem, and Dynamic Light Scattering. J Phys Chem B. 1998; 102: 6029-6039.

19) Binnig G, Quate CF, Gerber C. Atomic Force Microscope. Phys Rev Lett. 1986; 56: 930933.

20) Noy A, Sanders CH, Vezenov DV, Wong SS, Lieber CM. Chemically-Sensitive Imaging in Tapping Mode by Chemical Force Microscopy: Relationship between Phase Lag and Adhesion. Langmuir. 1998; 14: 1508-1511.

21) Burnham NA, Colton RJ, Pollock HM. Interpretation of Force Curves in Force Microscopy. Nanotechnology. 1993; 4: 64-80.

22) Abdelhady $\mathrm{HG}$, Allen $\mathrm{S}$, Davies $\mathrm{MC}$, Roberts CJ, Tendler SJB, Williams PM. Atomic Force Microscopy Studies of Generation 4 Poly(amidoamine) (PAMAM) Dendrimers on Functionalized Surfaces. Surf Sci. 2004; 558; 99 110.

23) Hierlemann A, Cambell JK, Baker LA, Crooks RM, Ricco, AJ. Structural Distortion of Dendrimers on Gold Surfaces: A Tapping Mode AFM Investigation. J Am Chem Soc. 1998; 120: 5323-5324.

24) Li J, Piehler T, Qin D, Baker JrJR, Tomalia DA. Visuallization and Characterization of Poly(amidoamine) Dendrimers by Atomic Microscopy. Langmuir. 2000;16: 5613-5626.
25) Li J, Swanson DR, Qin D, Brothers HM, Piehler LT, Tomalia D, Meier D J. Characterizations of Core-Shell Tecto- ( Dendrimer ) Molecules by Tapping Mode Atomic Force Microscopy. Langmuir. 1999; 15(21): 73477350.

26) Swanson SD, Kukowska-Latallo JF, Patri AK, Chen C, Ge S, Cao Z, Kotlyar A, et al. Targeted Gadolinium-Loaded Dendrimer Nanoparticles for Tumor-Specific Magnetic Resonance Contrast Enhancement. Int J Nanomedicine. 2008; 3(2): 201-210.

27) Wiener E, Settles M, Weirich G, Schmidt $C$, Diederichs G. The Influence of Collagen Network Integrity on the Accumulation of GadoliniumBased MR Contrast Agents in Articular Cartilage. Fortschr Röntgenstr. 2011; 183(3): 226-232.

28) Koper GJM, van Genderen MHP, ElissenRoma'n C, Baars MWPL, Meijer EW. and Borkovec M. Protonation Mechanism of Poly(propylene imine) Dendrimers and Some Associated Oligo Amines. J Am Chem Soc. 1997; 119: 6512-6521. 